\title{
SELF-INDUCED TRANSPARENCY BRAGG SOLITONS IN A NONLINEAR PHOTONIC BANDGAP STRUCTURE
}

\author{
P. RAMESH BABU* and K. SENTHILNATHAN \\ Photonics Division, Department of Physics, \\ School of Advanced Sciences, VIT University, \\ Vellore 632 014, Tamil Nadu, India \\ *prameshbabu@vit.ac.in
}

Received 19 January 2011

\begin{abstract}
We consider an optical pulse propagation in a one-dimensional nonlinear periodic structure doped uniformly with inhomogeneously broadening two-level atoms wherein the pulse propagation is governed by the nonlinear coupled mode-Maxwell Bloch equations. We investigate the pulse-train like periodic waves as well as bright and dark solitons near the photonic bandgap structure under the influence of self-induced transparency (SIT) effect. Further, we demonstrate that the resulting bright and dark SIT Bragg solitons may be realized both in anomalous and normal dispersion regimes, and this novel concept is ultimately the crux of this paper.
\end{abstract}

Keywords: Photonic bandgap; Self-induced transparency; soliton; fiber Bragg grating.

\section{Introduction}

It can be inferred from the literature that grating solitons are more advantageous than the conventional fiber solitons, owing to the fact that the former can be realized within a grating length of about a few cms. ${ }^{1-3}$ This is due to the fact that the grating induced dispersion is several orders of magnitude greater than the material dispersion. This paper deals with the impact of a phenomenon called self-induced transparency (SIT) that stems due to the doping of resonant atoms such as erbium, ytterbium, neodymium, and thulium etc. in the fiber Bragg grating (FBG) structure. SIT is essentially a phenomenon in which a short optical pulse does propagate practically with no loss through the optical media. This type of transparency takes place since each atom, even though sturdily excited, reradiates its excitation energy coherently in the forward direction, and this eventually induces a phase shift in the transmitted optical field. Further, in the SIT process, while the front part of the pulse excites the doped atoms of the resonant medium from the ground state to the upper state, the tail part of the pulse stimulates the active atoms from the upper state to the ground state. Owing to this atom-photon interaction, the SIT pulses can propagate through the medium with a group velocity that can be substantially lower than the speed of light in the host medium. ${ }^{4}$ With this effect, one can envision many possible applications such as delay line, pulse re-shaping, optical amplification, etc. 
As the aim of this paper is to investigate the generation of grating solitons in the presence of SIT effect, we consider the nonlinear pulse propagation in a FBG structure doped with the resonant atoms. In uniformly doped FBGs, the grating-induced dispersion balances with both the material Kerr nonlinearity and the resonant effects as determined by the Bloch equations. The resulting solitons are known as SIT Bragg solitons, which are essentially distortionless optical pulses. Distortionless SIT Bragg soliton pulses have been investigated in a uniformly doped nonlinear photonic bandgap (PBG) structure. ${ }^{5-9}$ Recently, Tseng and $\mathrm{Chi}^{8,9}$ investigated the existence of a moving SIT pulse train in a uniformly doped PBG structure and discussed the coexistence of SIT solitons and Bragg solitons. In this paper, we examine if these SIT Bragg solitons withstand the impact of the higher order perturbation effects. Besides, we study the generation of periodic waves which also satisfy both effective nonlinear Schrödinger (NLS) and Bloch equations.

\section{Theoretical Formulation of the Problem}

Here we consider a one-dimensional nonlinear periodic structure doped uniformly with the rare earth atoms. From Maxwell's equations, one can arrive at the following nonlinear coupled mode-Maxwell Bloch equations (NLCM-MB) that describe pulse propagation in a uniformly doped PBG structure ${ }^{8,9}$ :

$$
\begin{aligned}
& i \frac{\partial q_{f}}{\partial z}+i \beta_{1} \frac{\partial q_{f}}{\partial t}+\delta \beta_{0} q_{f}+\kappa q_{b}+\Gamma\left(\left|q_{f}\right|^{2}+2\left|q_{b}\right|^{2}\right) q_{f}+\frac{\mu_{0} \omega_{B}^{2}}{2 \beta_{g}}\left(P_{f}+\frac{2 i}{\omega_{B}} \frac{\partial P_{f}}{\partial t}\right)=0 \\
& -i \frac{\partial q_{b}}{\partial z}+i \beta_{1} \frac{\partial q_{b}}{\partial t}+\delta \beta_{0} q_{b}+\kappa q_{f}+\Gamma\left(\left|q_{b}\right|^{2}+2\left|q_{f}\right|^{2}\right) q_{b}+\frac{\mu_{0} \omega_{B}^{2}}{2 \beta_{g}}\left(P_{b}+\frac{2 i}{\omega_{B}} \frac{\partial P_{b}}{\partial t}\right)=0
\end{aligned}
$$

where $P_{f}$ and $P_{b}$ represent the slowly varying polarization envelopes induced by electric field envelopes $q_{f}$ and $q_{b}$, respectively. $\delta \beta_{0}=\beta_{0}-\beta_{g}$ implies the wave number detuning from the exact Bragg resonance, $\beta_{g}=\pi / \Lambda_{g}$ is the grating wave number, and $\Lambda_{g}$ is the grating period. The parameters $\beta_{0}$ and $\beta_{1}$ are determined by the mode propagation constant, $\beta(\omega) \equiv(\omega / c) n(\omega)$. Here, $\Gamma=n_{2} \omega_{B} /\left(c A_{\text {eff }}\right)$ is the Kerr nonlinear coefficient, $\omega_{B}=2 \pi c / \lambda_{B}$ is the Bragg frequency, $\lambda_{B}$ is the Bragg wavelength, $c$ is the velocity of light in vacuum, $n_{2}$ is the Kerr-nonlinear refractive index coefficient, and $A_{\text {eff }}$ is the effective core area. The parameters $\kappa$ and $\mu_{0}$ are the linear coupling coefficient and vacuum permeability, respectively. It can be noted that all the second order derivatives of $q_{f, b}$ and $P_{f, b}$ with respect to $z$ and $t$ have been neglected by using the slowly varying envelope approximation.

Following Refs. 8 and 9, we rewrite the above NLCM-MB equations and the resulting equations are called the effective NLCM equations which are as follows:

$$
\begin{gathered}
i \frac{\partial q_{f}}{\partial z}+i \beta_{1}^{e} \frac{\partial q_{f}}{\partial t}+\delta \beta_{e} q_{f}+\kappa q_{b}+\Gamma_{e}\left(\left|q_{f}\right|^{2}+2\left|q_{b}\right|^{2}\right) q_{f}=0 \\
-i \frac{\partial q_{b}}{\partial z}+i \beta_{1}^{e} \frac{\partial q_{b}}{\partial t}+\delta \beta_{e} q_{b}+\kappa q_{f}+\Gamma_{e}\left(\left|q_{b}\right|^{2}+2\left|q_{f}\right|^{2}\right) q_{b}=0
\end{gathered}
$$

where the effective parameters are $\delta \beta_{e}=\delta \beta_{0}+s c_{1} I_{1}-\left(2 s I_{1} / \omega_{B}\right)+s c_{2} I_{2}, \beta_{1}^{e}=\beta_{1}+$ $s c_{1} I_{1}+2 s c_{2} I_{2} / \omega_{B}, \Gamma_{e}=\Gamma+s c_{1} I_{1} \mu^{2} /\left(\omega_{B} \hbar^{2}\right)$. Here, $c_{1}$ and $c_{2}$ are the constants which 
relate to the frequency detuning and pulse width of the electric field, respectively. The parameter $s=\mu \mu_{0} \omega_{B}^{2} \omega_{i} /\left(2 \beta_{g} \hbar\right)$, where $\mu$ is the transition matrix element. $I_{1}$ and $I_{2}$ are the two integral constants which are given by $I_{1}=\int_{-\infty}^{\infty} f(\Delta \omega) g\left(\Delta \omega-\Delta \omega_{r 0}\right) d(\Delta \omega)$ and $I_{2}=$ $\int_{-\infty}^{\infty} \Delta \omega f(\Delta \omega) g\left(\Delta \omega-\Delta \omega_{r 0}\right) d(\Delta \omega)$. Here, $\Delta \omega=\omega_{r}-\omega_{B}, g\left(\Delta \omega-\Delta \omega_{r 0}\right)$ is the normalized inhomogeneous broadening line-shape function and similarly, $\Delta \omega_{r 0}=\omega_{r 0}-\omega_{B}$, and $\omega_{r 0}$ are the center of the broadening line-shape function.

From the above NLCM-MB equations and the effective NLCM equations, it could be seen that the nonlinear pulse propagation in a uniformly doped PBG structure may be regarded as an effective undoped PBG structure. It should be emphasized that the hitherto mentioned effective NLCM equations, in the hidden sense, do carry the essence of two-level system.

\section{Generation of SIT Bragg Solitons}

It is well-known that the SIT Bragg soliton is a distortionless optical pulse which results from the grating induced dispersion, balancing with both the material Kerr nonlinearity and the resonant effects being determined by the Bloch equations. Now, we apply the multiple scale analysis to examine the impact of the higher order perturbation effects near the PBG structure. In order to introduce the multiple scale analysis, we extend the linear solution in the following form:

$$
\left(\begin{array}{c}
q_{f} \\
q_{b}
\end{array}\right)=\varepsilon^{1 / 2} q\left(\tau_{1}, \tau_{2}, Z\right)\left(\begin{array}{c}
1 \\
-1
\end{array}\right) e^{-i \kappa t}+\varepsilon U_{1}+\varepsilon^{3 / 2} U_{2}+\varepsilon^{2} U_{3}+\cdots,
$$

where $\tau_{1}=\varepsilon t, \tau_{2}=\varepsilon^{2} t$, and $Z=\varepsilon^{1 / 2} z$. Here, $U_{1}, U_{2}, U_{3}$, etc. represent the perturbation parameters which indeed reflect both the linear and nonlinear higher order effects. Thus, the desired higher order effects can be studied by balancing the successive orders in $\varepsilon$ with the corresponding field envelopes. In order to investigate the impact of higher order perturbation effects, we continue balancing $O\left(\varepsilon^{2}\right)$ terms and this ultimately results in perturbed NLS equation which is given below:

$$
i \frac{\partial q}{\partial z_{1}}+A_{1} \frac{\partial^{2} q}{\partial t_{1}^{2}}+A_{2}|q|^{2} q+i A_{3} \frac{\partial^{3} q}{\partial t_{1}^{3}}+i A_{4}\left(|q|^{2} q\right)_{t_{1}}=0 .
$$

Here, the variables are $\tau_{1}=z_{1}$ and $Z=t_{1}$. In Eq. (4), $A_{1}, A_{2}, A_{3}$, and $A_{4}$ are the physical parameters of the NLCM equations in the nonlinear periodic structure which are defined as follows: $A_{1}=1 / 2 \kappa, A_{2}=3 \Gamma_{e}=3 \Gamma+3 s c_{1} I_{1} \mu^{2} /\left(\omega_{B} \hbar^{2}\right), A_{3}=1 / 8 \kappa^{3}$, and $A_{4}=3 \Gamma+3 s c_{1} I_{1} \mu^{2} /\left(\omega_{B} \hbar^{2}\right) / 4 \kappa^{2}$. This perturbed NLS equation represents the nonlinear pulse propagation in a periodic medium with higher order effects outside the PBG structure in a doped FBG. Now, we discuss the generation of bright soliton near the PBG structure by using the coupled amplitude-phase method. To proceed further, we express the field envelope, $q$, as,

$$
q\left(z_{1}, t_{1}\right)=Q\left(t_{1}+\beta z_{1}\right) \exp \left[i\left(k z_{1}-\omega t_{1}\right)\right], \quad \text { where } \chi=\left(t_{1}+\beta z_{1}\right) .
$$


Using Eq. (5) in (4) and comparing the resulting equation with Bloch equation, we get

$$
\left(\frac{d Q}{d \chi}\right)^{2}=\alpha Q^{2}-\frac{1}{2} \beta Q^{4}+\gamma
$$

where

$$
\alpha=\left(\frac{1}{c_{2}}-\frac{c_{1}}{4 c_{2}^{2}}\right)=\left(\frac{2 A_{1} \omega+3 A_{3} \omega^{2}-\beta_{1}}{A_{3}}\right), \quad \beta=\left(\frac{3 \mu^{2}}{4 \hbar^{2}}\right)=\left(\frac{A_{4}}{A_{3}}\right), \quad \gamma=C=\frac{4 c_{0}^{2}}{Q^{3}} .
$$

It should be pointed out that the Eq. (6) does have distortionless pulse-train solution i.e. cnoidal waves for $\gamma \neq 0$ as well as the single-pulse solution when $\gamma=0$. In this work, we discuss these two issues under the appropriate physical conditions. The choice that $\gamma=m^{2}\left(1-m^{2}\right) \alpha^{2} /\left(2 m^{2}-1\right)^{2} \beta$ yields the following cnoidal waves:

$$
q=\sqrt{p_{0}} c n\left[\frac{t-(z / V)}{T_{0}}, m\right] e^{i\left(k z_{1}-\omega t_{1}\right)},
$$

where the elliptic modulus is determined by the power, $p_{0}$, through the relation, $p_{0}=$ $2 m^{2} \alpha /\left(2 m^{2}-1\right) \beta$ and the period, $T_{0}$, is $\sqrt{2 m^{2} / p_{0} \beta}$.

According to the second physical condition, $\gamma=0$, we get the formation of bright soliton. It is to be noted that the same bright soliton pulse can also be obtained from Eq. (7) in the limit, $m=1$.

$$
q=\sqrt{2\left(\frac{2 A_{1} \omega+3 A_{3} \omega^{2}-\beta_{1}}{A_{4}}\right)} \operatorname{sech}\left(\sqrt{\left(\frac{2 A_{1} \omega+3 A_{3} \omega^{2}-\beta_{1}}{A_{3}}\right)}\right) \chi e^{i\left(k z_{1}-\omega t_{1}\right)}
$$

The soliton represented by the Eq. (8) has been formed near the frequency band edge due to interplay between positive nonlinearity, effective grating induced dispersion, and the resonant effects. Furthermore, the resulting soliton solution also satisfies the atomic Bloch equations. Consequently, this confirms the coexistence of a SIT soliton and a Bragg soliton. This kind of mixed state is referred to as a SIT-Bragg soliton. Figures (1a) and (1b) depict the intensity plots of $c n$ wave and bright SIT Bragg soliton, respectively.

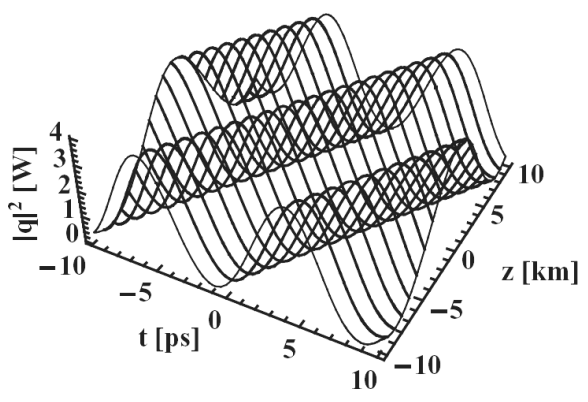

(a)

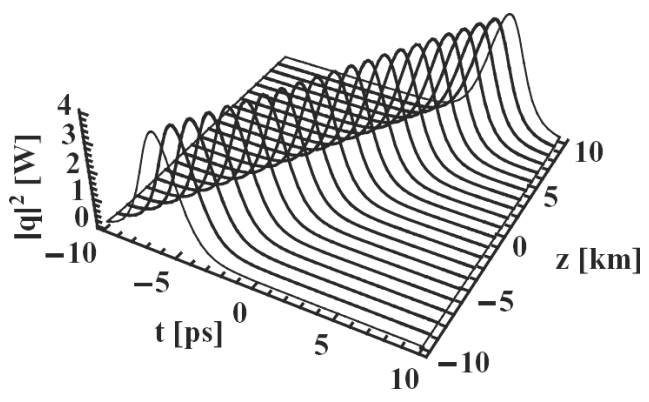

(b)

Fig. 1. (a) Intensity profile of the $c n(m=0.4)$ and (b) intensity profile of the bright $(m=1)$ SIT Bragg soliton, for $f=-1, p_{0}=2 \mathrm{~W}, k=12 \mathrm{~m}^{-1}, \omega=0.7 \mathrm{~Hz}$, and $V=0.4 \mathrm{~m} \mathrm{~s}^{-1}$. 
It is interesting to note that the soliton amplitude and the pulse width depend on the Kerr-coefficient and the dopant density of the two-level atoms. These factors distinguish the SIT-Bragg soliton from the conventional Bragg soliton. On the other hand, if the integration constant is chosen to be $\gamma=-(m \alpha)^{2} /\left(m^{2}+1\right)^{2} \beta$, Eq. (6) generates another exact periodic solution in terms of $s n$ as follows:

$$
q=\sqrt{p_{0}} s n\left[\frac{t-(z / V)}{T_{0}}, m\right] e^{i\left(k z_{1}-\omega t_{1}\right)}
$$

wherein the following conditions must be satisfied:

$$
p_{0}=\frac{2 m^{2} \alpha}{\left(m^{2}+1\right) \beta} \quad \text { and } \quad T_{0}=\sqrt{\frac{m^{2}+1}{\alpha}}
$$

As discussed earlier, based on the second physical condition $\gamma=0$, one can have the formation of dark SIT Bragg soliton in the limit, $m=1$, from Eq. (9).

$$
q=\sqrt{\left(\frac{2 A_{1} \omega+3 A_{3} \omega^{2}-\beta_{1}}{A_{4}}\right)} \tanh \left(\sqrt{\left(\frac{2 A_{1} \omega+3 A_{3} \omega^{2}-\beta_{1}}{2 A_{3}}\right)}\right) \chi e^{i\left(k z_{1}-\omega t_{1}\right)}
$$

Similarly, we can generate the cnoidal waves, as well as their limit forms, for $m=1$, which corresponds to the bright and dark SIT Bragg solitons in the case of the normal dispersion $\left(\beta_{2}^{g}>0\right)$ regime.

The intensity plots of $c n$ wave and dark SIT Bragg soliton are shown in Figs. (2a) and (2b), respectively. Our results describe that both bright and dark SIT Bragg solitons can be generated in the cases of the anomalous and normal dispersion regimes. This should be contrasted with the well-known situations in Refs. 1-3, where bright and dark SIT Bragg solitons exist, solely with the anomalous and normal dispersion regimes, respectively. In contrast to the fiber SIT soliton, SIT Bragg solitons can be realized experimentally, since uniformly doped FBGs have a length of only a few centimeters, owing to the large dispersion. This is long enough for generating SIT Bragg solitons.

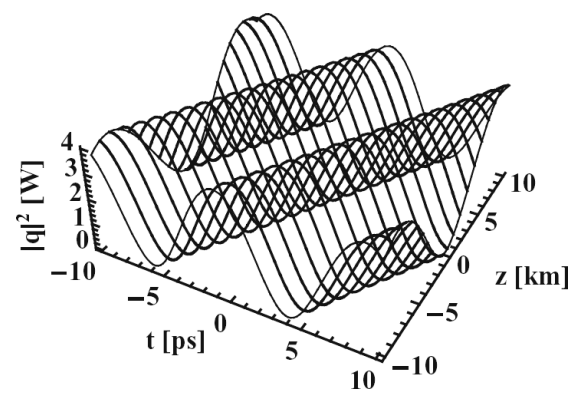

(a)

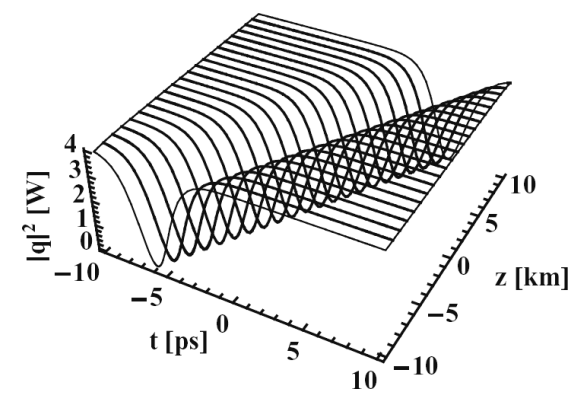

(b)

Fig. 2. (a) Intensity profile of the $s n(m=0.4)$ and (b) intensity profile of the dark $(m=1)$ SIT Bragg soliton, for $f=-1, p_{0}=2 \mathrm{~W}, k=12 \mathrm{~m}^{-1}, \omega=0.7 \mathrm{~Hz}$, and $V=0.4 \mathrm{~m} \mathrm{~s}^{-1}$. 


\section{Conclusion}

The generation of cnoidal waves, both $c n$ and sn types, as well as bright and dark SIT Bragg solitons has been delineated in a nonlinear PBG medium doped uniformly with inhomogeneously broadening two-level atoms. The results of this work bear a testimony to the very fact that both bright and dark SIT Bragg solitons can be realized in anomalous as well as normal dispersion regime. This is a novel concept in comparison with the wellknown situations in Refs. 1-3, wherein the existence of bright and dark SIT Bragg solitons has been reported only in the anomalous and normal dispersion regimes, respectively.

\section{Acknowledgments}

The authors are very much grateful for the encouragement and support rendered by the Management of VIT University, Vellore, 632 014, Tamil Nadu, India. One of the authors, K. Senthilnathan, would like to acknowledge the financial support through the research grant from Department of Science and Technology, Government of India.

\section{References}

1. C. M. de Sterke and J. E. Sipe, in Progress in Optics XXXIII, ed. E. Wolf (Elsevier, Amsterdam, 1994).

2. G. P. Agrawal, Applications of Nonlinear Fiber Optics (Academic Press, San Diego, 2001).

3. K. Senthilnathan, K. Porsezian, P. Ramesh Babu and V. Santhanam, Bright and dark Bragg solitons in a fiber Bragg grating, IEEE J. Quantum Elect. 39 (2003) 1192-1197.

4. Mc Call and Hahn, Self-Induced Transparency, Phys. Rev. 183 (1969) 457-485.

5. B. I. Mantsyzov, Gap $2 \pi$ pulse with an inhomogeneously broadened line and an oscillating solitary wave, Phys. Rev. A 51 (1995) 4939-4943.

6. N. Aközbek and S. John, Self-induced transparency solitary waves in a doped nonlinear photonic band gap material, Phys. Rev. E 58 (1998) 3876-3895.

7. G. Kurizki, A. E. Kozhekin, and B. A. Malomed, in Progress in Optics XLII, ed. E. Wolf (Elsevier, Amsterdam, 2001).

8. H. Y. Tseng and S. Chi, Distortionless pulse-train propagation in a nonlinear photonic band gap structure doped uniformly with inhomogeneously broadening two-level atoms, IEEE J. Sel. Top. Quantum Electron. 8 (2002) 681-689.

9. H. Y. Tseng and S. Chi, Coexistence of a self-induced transparency soliton and a Bragg soliton, Phys. Rev. E 66 (2002) 056606. 